\title{
Expression of the chemokine receptor CCR1 promotes the dissemination of multiple myeloma plasma cells in vivo
}

Haematologica 2021

Volume 106(12):3176-3187

\section{Correspondence:}

KATE VANDYKE

kate.vandyke@adelaide.edu.au

Received: March 25, 2020.

Accepted: October 23, 2020.

Pre-published: November 5, 2020.

https://doi.org/10.3324/haematol.2020.253526

\section{(C)2021 Ferrata Storti Foundation}

Material published in Haematologica is covered by copyright. All rights are reserved to the Ferrata Storti Foundation. Use of published material is allowed under the following terms and conditions:

https://creativecommons.org/licenses/by-nc/4.0/legalcode. Copies of published material are allowed for personal or internal use. Sharing published material for non-commercial purposes is subject to the following conditions:

https://creativecommons.org/licenses/by-nc/4.0/legalcode, sect. 3. Reproducing and sharing published material for commercial purposes is not allowed without permission in writing from the publisher.

\author{
Mara N. Zeissig, ${ }^{1,2}$ Duncan R. Hewett, ${ }^{1,2}$ Vasilios Panagopoulos, ${ }^{1,2}$ Krzysztof M. \\ Mrozik, ${ }^{1,2}$ L. Bik To, ${ }^{3}$ Peter I. Croucher, ${ }^{4,5}$ Andrew C.W. Zannettino ${ }^{1,2,6,7 \#}$ and Kate \\ Vandyke $^{1,2 \#}$
}

${ }^{1}$ Myeloma Research Laboratory, Adelaide Medical School, Faculty of Health and Medical Sciences, University of Adelaide, Adelaide, South Australia; ${ }^{2}$ Precision Medicine Theme, South Australian Health and Medical Research Institute, Adelaide, South Australia; ${ }^{3}$ Department of Hematology, Royal Adelaide Hospital, Adelaide, South Australia; ${ }^{4}$ Bone Biology Division, Garvan Institute of Medical Research, Sydney, New South Wales; ${ }^{5} \mathrm{St}$ Vincent's Clinical School, Faculty of Medicine, University of New South Wales, Sydney, New South Wales; ${ }^{6}$ Central Adelaide Local Health Network, Adelaide, New South Wales and ${ }^{7}$ Center for Cancer Biology, University of South Australia, Adelaide, New South Wales, Australia.

${ }^{\#} A C W Z$ and $K V$ contributed equally as co-senior authors.

\section{ABSTRACT}

M ultiple myeloma (MM) disease progression is dependent on the ability of MM plasma cells (PC) to egress from the bone marrow (BM), enter the circulation and disseminate to distal BM sites. Expression of the chemokine CXCL12 by BM stromal cells is crucial for MM PC retention within the BM. However, the mechanisms which overcome CXCL12-mediated retention to enable dissemination are poorly understood. We have previously identified that treatment with the CCR1 ligand CCL3 inhibits the response to CXCL12 in MM cell lines, suggesting that CCL3/CCR1 signaling may enable egress of MM PC from the BM. Here, we demonstrated that CCR1 expression was an independent prognostic indicator in newly diagnosed $\mathrm{MM}$ patients. Furthermore, we showed that CCR1 is a crucial driver of dissemination in vivo, with CCR1 expression in the murine MM cell line 5TGM1 being associated with an increased incidence of bone and splenic disseminated tumors in C57BL/KaLwRij mice. Furthermore, we demonstrated that CCR1 knockout in the human myeloma cell line OPM2 resulted in a $>95 \%$ reduction in circulating MM PC numbers and BM and splenic tumor dissemination following intratibial injection in NSG mice. Therapeutic targeting of CCR1 with the inhibitor CCX9588 significantly reduced OPM2 or RPMI-8226 dissemination in intratibial xenograft models. Collectively, our findings suggest a novel role for CCR1 as a critical driver of BM egress of MM PC during tumor dissemination. Furthermore, these data suggest that CCR1 may represent a potential therapeutic target for the prevention of $\mathrm{MM}$ tumor dissemination.

\section{Introduction}

Multiple myeloma (MM) is an incurable hematological cancer characterized by the uncontrolled proliferation of clonal plasma cells $(\mathrm{PC})$ within the bone marrow (BM). ${ }^{1}$ One of the key features of $\mathrm{MM}$ is the presence of MM PC at multiple sites throughout the BM, highlighting that dissemination of transformed PC is a critical process during disease development. ${ }^{1,2}$ In support of this, circulating MM PC are detectable by flow cytometry in approximately $75 \%$ of newly diagnosed $\mathrm{MM}$ patients. ${ }^{3}$ Importantly, the presence of elevated circulating MM PC predicts faster time to progression and poorer overall survival, independent of BM tumor burden. ${ }^{4-12}$

The dissemination of MM PC is a multi-step process requiring release from the supportive niche in the BM, intravasation into nearby blood vessels and subsequent 
extravasation and homing to another BM site. Integrin mediated adhesion of MM PC to BM stromal cells (BMSC), and extracellular matrix components synthesized by BMSC, is well-established to mediate retention of MM PC within the niche. ${ }^{13}$ For example, MM PC express the integrin $\alpha 4 \beta 1$ (also known as very late antigen 4 , VLA-4) that mediates adhesion to vascular cell-adhesion molecule 1 (VCAM-1) on BMSC and to the extracellular matrix component fibronectin. ${ }^{13}$ Importantly, the C-X-C chemokine ligand CXCL12 (also known as stromal cell-derived factor1; SDF-1), abundantly produced by BMSC, ${ }^{14}$ enhances adhesion to fibronectin and VCAM-1 through binding to its receptor CXCR4 on the surface of MM PC and inducing rapid conformational changes of the integrin $\alpha 4 \beta 1 \mathrm{com}-$ plex on MM PC. ${ }^{15}$ Notably, plerixafor-mediated inhibition of the CXCL12 receptor CXCR4 on MM PC results in mobilization of $\mathrm{MM}$ cells to the peripheral blood (PB) in a preclinical model of $\mathrm{MM}^{15}$ These data suggest that CXCL12 is a critical BM retention signal for MM PC and that overcoming the CXCL12/CXCR4 signal may be required for release from the niche during dissemination.

In a previous study by Azab and colleagues, increased hypoxia in the BM was shown to be associated with an increase in circulating MM PC in a preclinical model. ${ }^{16}$ Additionally, we have previously identified that overexpression of the hypoxia-inducible factor $2 \alpha$ (HIF-2 $\alpha$ ) in $\mathrm{MM}$ cell lines reduces their response to exogenous CXCL12 in vitro, suggesting that hypoxia may overcome CXCL12-mediated retention. Furthermore, we identified that hypoxia and HIF- $2 \alpha$ increased expression of the C-C chemokine receptor CCR1 in human MM cell lines. ${ }^{17}$ CCR1 is a seven-transmembrane G-protein coupled receptor and its most potent activator is CCL3 (also known as macrophage inflammatory protein $1 \alpha$; MIP-1 $1 \alpha$ ). Previous literature suggests that MM PC abundantly produce CCL3 ${ }^{18-21}$ which activates CCR1 expressed on osteoclasts leading to increased osteolysis, ${ }^{19}$ with CCR1 antagonists reducing osteolysis in a murine model of $\mathrm{MM}^{22,23}$ In addition, CCL3 is a potent inducer of migration of patientderived MM PC and MM cell lines in vitro. ${ }^{17,19,20,24}$ In hematopoietic progenitors and natural killer cells, CCL3/CCR1 signaling drives mobilization from the BM, in part by inactivation of CXCL12/CXCR 4..$^{25,26}$ Similarly, our previous studies showed that either pre-treatment of $\mathrm{MM}$ cell lines with CCL3 or elevated CCR1 expression decreased tumor cell migration towards CXCL12 in vitro. ${ }^{17}$ Taken together, these data suggest that hypoxia-mediated increases in CCR1 expression may desensitize cells to CXCL12-mediated BM retention and thereby facilitate dissemination. In support of this, we have previously shown that expression of CCR1 in MM PC is associated with poorer prognosis and an increase in the number of circulating MM PC in newly diagnosed MM patients. ${ }^{17}$ Here, we further investigated the association between CCR1 expression and poor overall survival rates in $\mathrm{MM}$ patients. Furthermore, we investigated the role for CCR1 in the dissemination of MM PC in vivo. Initially, we determined whether CCR1 overexpression can promote tumor dissemination in the syngeneic $5 \mathrm{TGM} 1 / \mathrm{KaLwRij}$ murine model of MM. Furthermore, using xenograft models of MM, we assessed whether CCR1 knockout limits the dissemination of MM PC in vivo. Lastly, we investigated whether pharmacological inhibition of CCR1 can be used as a viable therapeutic strategy to limit MM PC dissemination.

\section{Methods}

\section{Flow cytometry on patient samples}

Ethical approval for this study was obtained from the University of Freiburg Medical Center Ethics Review Committee and all patients provided written, informed consent, in accordance with the Declaration of Helsinki. CCR1 analysis was conducted on $\mathrm{BM}$ mononuclear cells from BM aspirates from 28 newly diagnosed MM (median age: 68 years [range, 49-84]; male:female ratio 1.15:1) and seven monoclonal gammopathy of undetermined significance (MGUS) ${ }^{1}$ (median age: 74 years [range, 53-88]; male:female ratio 1.7:1) patients. Cell surface CCR1 expression was assessed on viable $\mathrm{CD} 38^{++} / \mathrm{CD} 138^{+} / \mathrm{CD} 45^{\circ} / \mathrm{CD} 19$ malignant PC by multicolor flow cytometry (FACSARIA III; BD Biosciences, San Jose, CA) as previously described. ${ }^{17}$

\section{Murine multiple myeloma models models}

C57BL.KaLwRijHsd (KaLwRij) mice were inoculated into the left tibia with $1 \times 10^{5}$ 5TGM1-CCR1 or 5TGM1-EV cells. After 25 days, splenic tumor burden was assessed by bioluminescent imaging (Xenogen IVIS 100; Perkin Elmer), and tumor burden in the PB, injected tibiae, and pooled tibiae and femora from the contralateral leg was assessed by flow cytometry (LSRFortessa flow cytometer).

NOD.Cg-Prkdc ${ }^{\text {scid }} I 12 \mathrm{rg}^{\mathrm{m} 1 \mathrm{~W}_{\mathrm{j}} /} / \mathrm{SzJ}$ (NSG) mice were inoculated intratibially with $5 \times 10^{5}$ OPM2-CCR1-KO-1 or OPM2-EV-1 cells. For CCX9588 studies, mice were treated at 12-hour intervals via oral gavage with either the CCR1 antagonist CCX9588 $(15 \mathrm{mg} / \mathrm{kg}$; ChemoCentryx, CA) or polyethylene glycol (PEG) vehicle alone, commencing day 3 or day 14 following tumor cell injection. Primary and secondary BM and splenic tumor burden and $\mathrm{PB}$ tumour cells were assessed 28 days after tumor cell injection.

Detailed methods can be found in the Online Supplementary Methods.

\section{Results}

\section{High CCR1 expression is associated with poorer prognosis in multiple myeloma patients}

We used flow cytometry to examine the expression of CCR 1 on $\mathrm{CD}^{\circ} 8^{++} / \mathrm{CD} 138^{+} / \mathrm{CD} 45^{\mathrm{lo}} / \mathrm{CD} 19^{-} \mathrm{BM} \mathrm{PC}$ in a cohort of MM and MGUS patients who had not received previous treatment. BM PC expression of CCR1 was detectable by flow cytometry in $14.3 \%$ (one of seven) of MGUS patients and in $53.6 \%$ (15 of 28) of MM patients (Figure 1A). Furthermore, BM PC expression of CCR1 was significantly higher in MM patients than in MGUS patients $(P<0.05$, Figure $1 \mathrm{~A})$, consistent with our previous microarray analysis. ${ }^{17}$

Our previous analysis suggested that high levels of CCR1 expression in BM PC from newly diagnosed MM patients was associated with poorer overall survival in patients enrolled in the total therapy 3 trial. ${ }^{17}$ Here, we performed Cox regression analysis to determine if elevated (above median) CCR1 expression was an independent predictor of poor prognosis. In univariate analyses, elevated CCR 1 expression, high-risk gene expression signature, elevated serum $\beta 2$ microglobulin ( $\geq 5.5 \mathrm{mg} / \mathrm{L}$ ), anemia (hemoglobin $<$ $100 \mathrm{mg} / \mathrm{L}$ ) and high plasma cell proliferative index (PI $\geq 10 \%)$ were associated with inferior overall survival $(P<0.05$, Table 1$)$. Notably, multivariable analysis demonstrated that elevated $C C R 1$ retained its association with poor prognosis $(P<0.05$, hazard ratio $[\mathrm{HR}]=2.5,95 \%$ confidence interval $[\mathrm{CI}]:$ 1.0-5.9), when these other prognostic 
factors were taken into account (Table 1). In order to further investigate if CCR1 expression was associated with poor outcomes for MM patients, we assessed CCR1 expression in BM PC using RNA-sequencing data from a cohort of MM patients who had a sample taken at diagnosis (baseline) and a sample taken following at least one line of therapy (subsequent). These data suggested that patients with relatively high CCR 1 expression at baseline $(\mathrm{n}=7 ; P<0.05, \mathrm{HR}=4.3$, 95\% CI: 1.0-18.1) or patients with elevated CCR 1 expression following treatment $(\mathrm{n}=10 ; P=0.080, \mathrm{HR}=3.0,95 \% \mathrm{CI}$ : 0.9-10.4) tended to have inferior survival compared with patients with low CCR1 expression both at baseline and following therapy ( $\mathrm{n}=26$; Figure $1 \mathrm{~B}$ and $\mathrm{C})$. Taken together, these data suggest that CCR1 expression either at baseline or following treatment may be associated with poorer overall survival for MM patients.

\section{Expression of CCR1 in the mouse multiple myeloma cell line 5TGM1 does not affect proliferation in vitro and increases incidence of splenic and bone dissemi- nation in vivo}

As we have previously shown that CCR1 expression is associated with increased circulating MM PC numbers in MM patients, ${ }^{17}$ we hypothesized that the association between increased CCR1 expression and poor prognosis was due to a role for CCR1 in MM PC dissemination. In order to investigate this, we initially assessed whether constitutive expression of CCR1 affected the migration and dissemination of the mouse MM cell line 5TGM1, which does not express detectable CCR1 basally (Figure 2A), and exhibits low levels of spontaneous dissemination in vivo. ${ }^{29}$ Expression of functional HA-tagged CCR 1 was confirmed by quantitative polymerase chain reaction (PCR) and by immunoprecipitation/western blotting (Figure 2A and B) and by the ability of the 5TGM1-CCR1 cells to migrate towards recombinant human CCL3 (rhCCL3) in a transwell assay (Figure 2C). Expression of CCR1 did not affect the proliferation of 5TGM1 cells, relative to 5TGM1-EV controls, either basally $(P=0.63$; Figure $2 \mathrm{D})$ or following addition of rhCCL3 ( $P=0.99$; Figure $2 \mathrm{E})$.

In order to investigate whether CCR1 expression affects 5TGM1 dissemination in vivo, 5TGM1-CCR1 or 5TGM1EV cells were intratibially injected into C57BL/KaLwRij mice. Primary tumor burden in the injected tibiae was not significantly different between animals injected with 5TGM1-CCR1 cells and controls $(P=0.82$; Figure 3A). Similarly, the numbers of circulating MM cells in the $\mathrm{PB}$, or the tumor burden in the contralateral leg, were also not significantly different between groups $(P=0.62$ and $P=0.41$, respectively; Figure $3 \mathrm{~B}$ and $\mathrm{C}$ ). However, there was a significant increase in the incidence of tumor in the 5TGM1CCR1 group, with eight of 11 mice $(73 \%)$ in this group having detectable green fluorescence protein positive $\left(\mathrm{GFP}^{+}\right)$ cells in the contralateral leg, compared with four of 11 mice (36\%) injected with 5TGM1-EV cells ( $P<0.0001$; Figure 3D). Furthermore, an increase in the incidence of dissemination to the spleen was also observed in the 5TGM1-CCR1 group, with nine of 11 mice $(82 \%)$ having tumors detectable in the spleen by bioluminescence imaging, compared with four of eight mice $(50 \%)$ in the 5TGM1-EV cohort $(P<0.0001$; Figure $3 \mathrm{E}$ and $\mathrm{F})$. Collectively, these data suggest that expression of CCR1 increases dissemination of MM PC, without affecting primary tumor growth.

Knockout of CCR1 in the human multiple myeloma cell line 0PM2 does not affect proliferation in vitro and prevents dissemination in vivo

In order to further investigate the role of CCR1 in tumor dissemination in $\mathrm{MM}$, we generated CRISPR/Cas9-mediated CCR1 knockouts (KO) in the
A

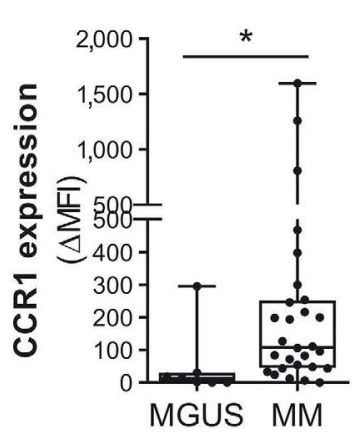

C

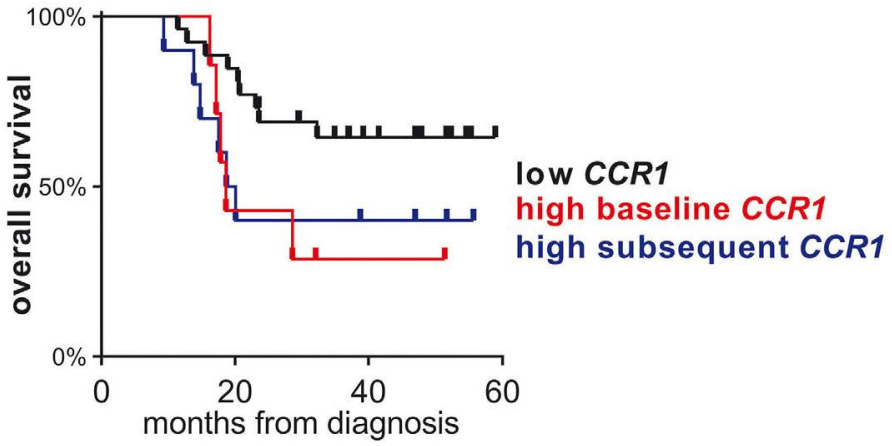

B

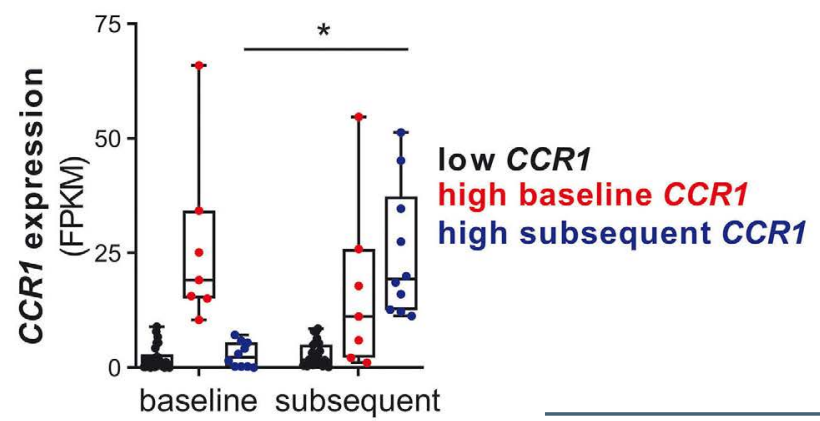

Figure 1. CCR1 is expression is elevated in multiple myeloma patients and is associated with poor prognosis. (A) CCR1 expression $(\triangle \mathrm{MFI})$ on $\mathrm{CD} 38^{++} / \mathrm{CD} 138^{+} / \mathrm{CD} 45^{10} / \mathrm{CD} 19$ bone marrow (BM) plasma cells (PC) from newly diagnosed monoclonal gammopathy of undetermined significance (MGUS) $(n=7)$ and multiple myeloma (MM) $(n=28)$ patients was assessed by flow cytometry. Graph depicts median with interquartile range, showing all data points. (B) CCR1 expression is shown for CD138-selected BM MM PC from patients with a sample taken at diagnosis (baseline) and a sample taken following at least one line of therapy with bortezomib (subsequent) (CoMMpass RNA-sequencing dataset, $n=43$ patients). Patients were categorized as having low tumor expression of CCR1 (CCR1 <10 FPKM at both baseline and subsequent biopsy; $\mathrm{n}=26$ ), high CCR1 (CCR1 $\geq 10 \mathrm{FPKM}$ at baseline; $n=7$ ) or increased CCR1 (baseline CCR1 <10 FPKM and subsequent CCR1 $\geq 10$ FPKM; $n=10$ ). (C) Kaplan-Meier plots of overall survival are shown for MM patients stratified based on BM MM PC expression of CCR1 at baseline and subsequent to therapy. 
human MM cell line OPM2 (Online Supplementary Figure S1). Loss of CCR1 protein expression in OPM2-CCR1KO-1 and OPM2-CCR1-KO-2 cell lines was confirmed by flow cytometry (Figure 4A). Furthermore, migration towards rhCCL3 was not observed in the OPM2-CCR1$\mathrm{KO}$ cell lines, confirming loss of CCR1 function (Figure 4B). Proliferation of OPM2 cell lines was unaffected by
CCR1 KO, either basally (Figure 4C and D) or following addition of rhCCL3 (Figure 4E).

In order to determine if CCR1 KO limited MM PC dissemination in vivo, NSG mice were injected with either OPM2-EV-1 or OPM2-CCR1-KO-1 cells. KO of CCR1 reduced primary tumor burden by $45.5 \%$, compared with controls (Figure 5A). Circulating tumor cell numbers were

Table 1. Univariate and multivariable analysis of factors associated with overall survival in multiple myeloma patients. ${ }^{1}$

\begin{tabular}{|c|c|c|c|c|c|}
\hline & \multirow[b]{2}{*}{$\begin{array}{l}n \\
(\%)\end{array}$} & \multicolumn{2}{|c|}{ Univariate analysis } & \multicolumn{2}{|c|}{ Multivariable analysis } \\
\hline & & P-value ${ }^{2}$ & $\begin{array}{c}H^{3} \\
(95 \% \text { Cl) }\end{array}$ & P-value ${ }^{2}$ & $\begin{array}{c}H R^{3} \\
(95 \% \text { Cl) }\end{array}$ \\
\hline$C C R 1>$ median & $\begin{array}{c}71 \\
(50 \%)\end{array}$ & 0.026 & $\begin{array}{c}2.46 \\
(1.11-5.45)\end{array}$ & 0.039 & $\begin{array}{c}2.48 \\
(1.05-5.86)\end{array}$ \\
\hline High-risk gene signature $^{4}$ & $\begin{array}{c}38 \\
(26.8 \%)\end{array}$ & 0.044 & $\begin{array}{c}2.16 \\
(1.02-4.57)\end{array}$ & 0.50 & $\begin{array}{c}1.36 \\
(0.56-3.27)\end{array}$ \\
\hline Age $\geq 65$ years & $\begin{array}{c}36 \\
(25.4 \%)\end{array}$ & 0.62 & $\begin{array}{c}0.77 \\
(0.32-1.97)\end{array}$ & - & - \\
\hline$\beta 2$-microglobulin $\geq 5.5 \mathrm{mg} / \mathrm{L}$ & $\begin{array}{c}33 \\
(23.2 \%)\end{array}$ & 0.021 & $\begin{array}{c}2.45 \\
(1.15-5.24)\end{array}$ & 0.60 & $\begin{array}{c}1.26 \\
(0.53-3.00)\end{array}$ \\
\hline Albumin $<35 \mathrm{~g} / \mathrm{L}$ & $\begin{array}{c}31 \\
(21.8 \%)\end{array}$ & 0.54 & $\begin{array}{c}1.31 \\
(0.56-3.08)\end{array}$ & - & - \\
\hline Hemoglobin $<100 \mathrm{~g} / \mathrm{L}$ & $\begin{array}{c}40 \\
(28.2 \%)\end{array}$ & 0.001 & $\begin{array}{c}3.52 \\
(1.67-7.45)\end{array}$ & 0.009 & $\begin{array}{c}3.18 \\
(1.34-7.56)\end{array}$ \\
\hline Proliferative index $\geq 10 \%$ & $\begin{array}{c}20 \\
(14.1 \%)\end{array}$ & 0.018 & $\begin{array}{c}2.69 \\
(1.18-6.12)\end{array}$ & $\begin{array}{c}0.61 \\
(0.48-3.44)\end{array}$ & 1.29 \\
\hline
\end{tabular}

${ }^{1}$ Gene expression analysis and clinical data from $\mathrm{n}=142$ newly diagnosed multiple myeloma patients in the total therapy 3 (TT3) trial (E-TABM-1138),28 ${ }^{28}$ Cox proportional hazards models; ${ }^{3} \mathrm{Hazard}$ ratio (HR); ${ }^{4} \mathrm{MS}$, MF or PR gene-expression profiling-defined subgroups $;{ }^{48} \mathrm{CI}$ : confidence interval.

A

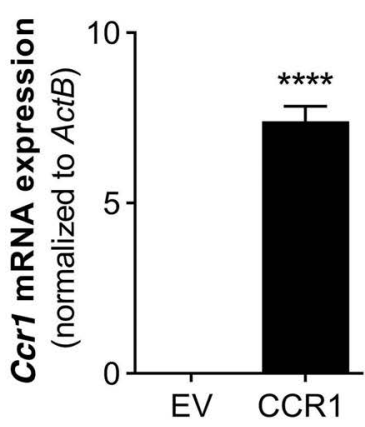

D

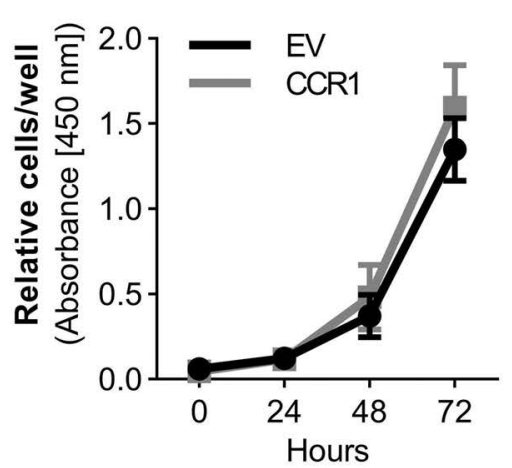

B



IP: HA

WB: HA
C

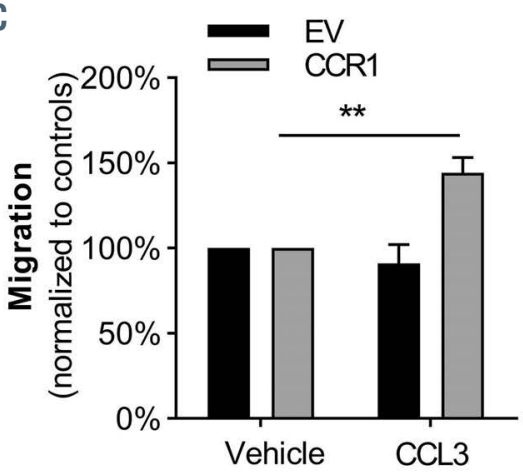

E

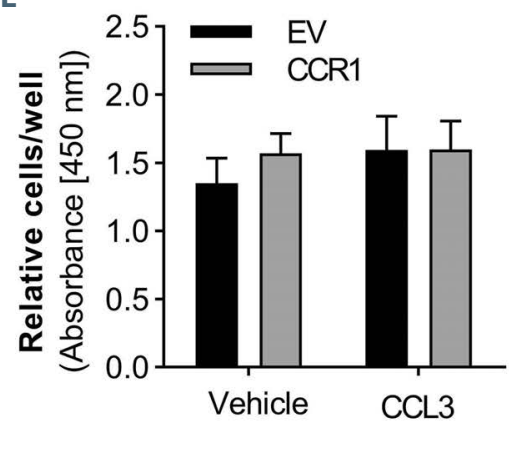

Figure 2. CCR1 expression in 5TGM1 murine multipe myeloma cell line increases migration towards CCL3 but does not affect proliferation. (A) Expression of murine Ccr1 mRNA was confirmed in 5TGM1-CCR1 cells. (B) CCR1-HA protein expression in 5TGM1-CCR1 cells was confirmed by immunoprecipitation using an anti-HA antibody followed by western blotting with anti-HA antibody. A representative of two independent experiments is shown. (C) Migration of 5TGM1-CCR1 and empty vector control (EV) cells towards $100 \mathrm{ng} / \mathrm{mL}$ rhCCL3 was assessed after 24 hours. (D) Relative number of 5TGM1-CCR1 and -EV cells was assessed over 72 hours. (E) Relative number of 5TGM1-CCR1 and -EV cells was assessed following 72 hours of culture with or without addition of $100 \mathrm{ng} / \mathrm{mL}$ rhCCL3. Graphs depict mean \pm standard error of the mean of three biological replicates (A) or three or more independent experiments (C to $E)$. $* * P<0.01$, ****P<0.0001, unpaired $t$-test (A), twoway ANOVA with Sidak's multiple comparison test (C). 
reduced by $97.8 \%$ in mice bearing CCR1 KO cells compared with controls $(P<0.0001$; Figure $5 \mathrm{~B})$. Additionally, dissemination of OPM2 cells to the contralateral leg was not observed in mice bearing OPM2-CCR1-KO-1 cells, with a $99.9 \%$ reduction in BM disseminated tumor cells compared with empty vector (EV) controls $(P<0.0001$; Figure 5C). Similar results were seen in the development of splenic dissemination, with mice inoculated with OPM2-EV-1 cells developing splenomegaly (Figure 5D) resulting from tumor cell infiltration, as confirmed by immunohistochemistry for $\mathrm{GFP}^{+}$cells (Figure 5E), which was markedly reduced in mice inoculated with OPM2CCR1-KO-1 cells $(P<0.0001$; Figure 5D).

We have previously demonstrated that CCL3 binding to CCR1 completely abrogates MM PC response to exogenous CXCL12 in vitro, without affecting CXCR4 expression, ${ }^{17}$ suggesting a mechanism whereby increased CCR1 expression may enable the dissemination of MM PC from the BM. We therefore hypothesized that CCR $1 \mathrm{KO}$ cell lines may retain their response to BM CXCL12, thereby leading to retention within the BM niche. Consistent with our previous data, pre-treatment of OPM2-EV-1 cells with CCL3 prevented their migration towards CXCL12 (Online Supplementary Figure S2). In contrast, OPM2-CCR1-KO-1 cells retained their ability to migrate in response to exogenous CXCL12, even in the presence of CCL3 (Online Supplementary Figure S2). CCR1 KO had no effect on the expression of CXCR4 or CXCL12 in OPM2 cells (Online Supplementary Figure S3), consistent with our previous findings. ${ }^{17}$ In order to investigate the mechanism whereby CCR1 loss abrogates the dissemination of OPM2 cells in vivo, we assessed whether CCR1 KO had a compensatory effect on the expression of other factors that are known to play a role in MM PC adhesion and migration. CCR $1 \mathrm{KO}$ in OPM2 cells did not lead to a compensatory expression of the alternate CCL3 receptor CCR5, nor did it affect expres-
A

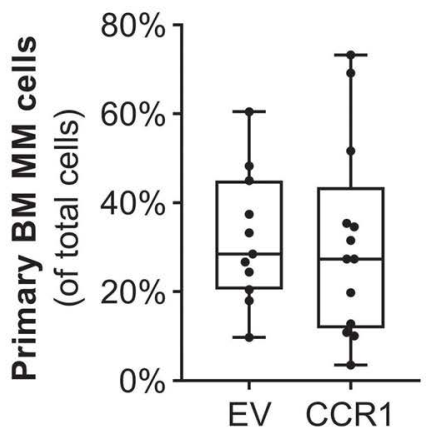

C

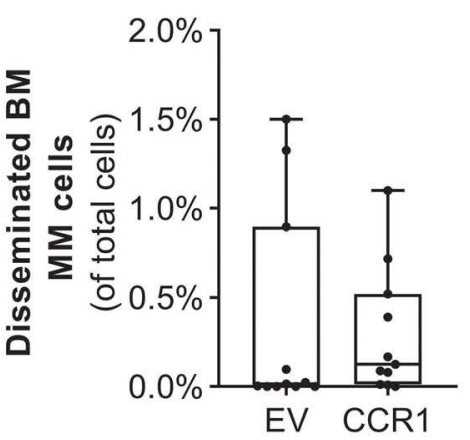

$E$

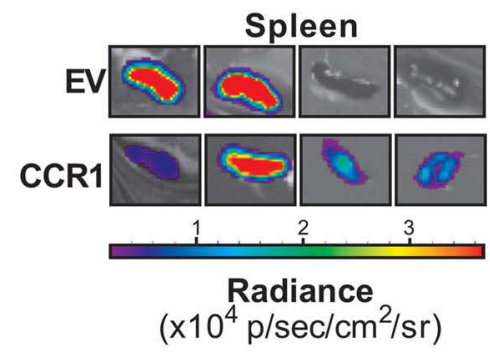

B

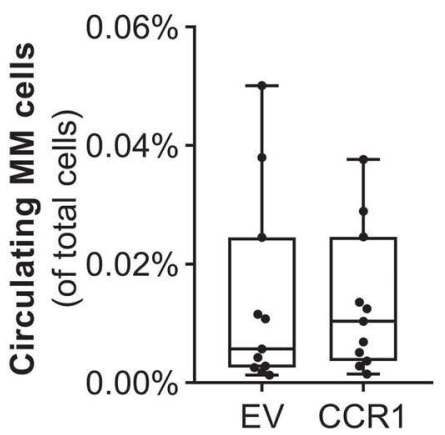

D

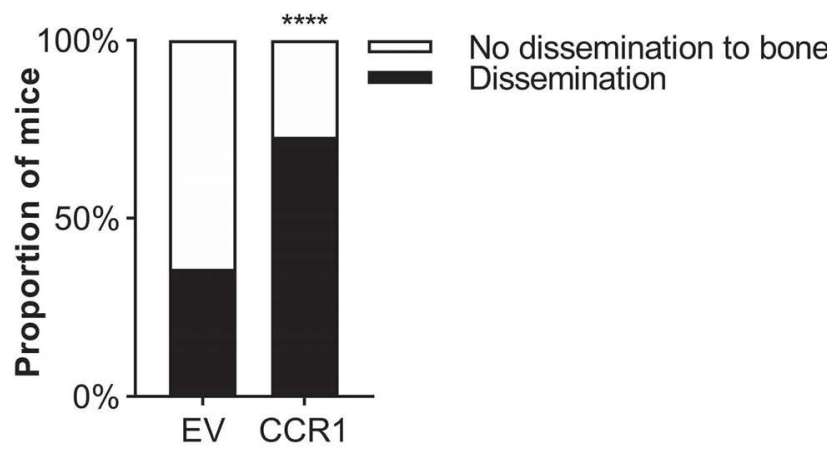

$\mathbf{F}$

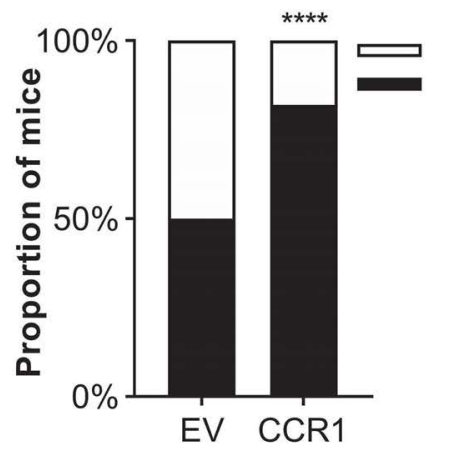

No dissemination to spleen Dissemination

Figure 3. CCR1 expression in 5TGM1 multiple myeloma plasma cells increases incidence of bone and splenic dissemination in a C57BL/KaLwRij intratibial mode of MM. (A) Primary tumor burden in injected tibiae after 3.5 weeks in C57BL/KaLwRij mice injected with 5TGM1-CCR1 or control 5TGM1-EV cells. Percentage of green fluorescence protein positive (GFP+) multiple myeloma (MM) cells of total mononuclear cells were quantitated using flow cytometry. (B) Number of circulating 5TGM1CCR1 or -EV cells in peripheral blood of mice. (C) Tumor burden disseminated to the non-injected contralateral leg in mice injected with 5TGM1-CCR1 or -EV cells. (D) Proportion of mice with detectable GFP+ MM cells in the contralateral long bones. (E) Spleens were collected from eight mice (5TGM1-EV) and 11 mice (5TGM1CCR1) and imaged using bioluminescence imaging, with representative spleens from each group shown. (F) Proportion of mice with detectable bioluminescence signal in the spleen. Box and whisker plots depict median and interquartile range for 11 mice/group (A to C). $* * * * P<0.0001$, Fisher's exact test. EV: empty vector. 
sion of integrin $\alpha 4$ (ITGA4) and integrin $\beta 1$ (ITGB1), critical adhesion molecules that play a role in MM PC BM retention (Online Supplementary Figure S3).

\section{The CCR1 inhibitor CCX9588 inhibits migration towards CCL3 in vitro}

Next, the effects of a selective small molecule CCR1 inhibitor, CCX9588, on MM cells was assessed in vitro. In order to investigate whether the small molecule CCR1 inhibitor CCX9588 effects cell survival and/or proliferation, CCR1-expressing OPM2-EV-1 or RPMI-8226-luc ${ }^{17}$ cells were cultured with increasing concentrations of CCX9588 or vehicle alone. OPM2-EV-1 cell number $(P=0.88$, Figure $6 \mathrm{~A})$ and viability $(P=0.70$, Figure $6 \mathrm{~B})$ were not affected by treatment with up to $1 \mu \mathrm{M}$ CCX9588. However, there was a 35\% decrease in cell number in RPMI-8226-luc cells treated with $1 \mu \mathrm{M}$ CCX9588 $(P<0.01$, Figure 6C), while cell survival was unaffected $(P=0.50$, Figure $6 \mathrm{D})$, suggesting that high concentrations may decrease proliferation of these cells. Based on these results, concentrations up to $100 \mathrm{nM}$ and $1 \mu \mathrm{M}$ were used for further characterization in RPMI8226 and OPM2 cells, respectively.

In order to confirm the anti-CCR1 function of CCX9588, OPM2-EV-1 or RPMI-8226-luc cells were treated with CCX9588 or vehicle alone and were then stimulated with rhCCL3. Western blot analysis revealed that, in OPM2-EV1 cells, CCL3 treatment induced AKT and ERK1/2 phosphorylation, which was inhibited by $10 \mathrm{nM}$ CCX9588 or higher (Figure 6E). In RPMI-8226-luc cells, AKT phosphorylation was increased by CCL3 and this was inhibited by 10 nM CCX9588 or higher (Figure 6F). Furthermore, pre-treat- ment of OPM2 or RPMI-8226 cells with CCX9588 resulted in a complete inhibition of migration towards rhCCL3 in a transwell assay (OPM2: $P<0.001$, Figure 6G; RPMI-8226: $P<0.01$, Figure $6 \mathrm{H})$.

\section{CCX9588 treatment reduces dissemination of multiple} myeloma plasma cells in vivo

In order to investigate the effectiveness of CCR1 inhibition in suppressing MM PC dissemination in vivo, the effects of the CCR1 inhibitor CCX9588 were assessed in mice bearing OPM2-EV-1 or RPMI-8226-luc tumors. CCX9588 treatment did not have appreciable adverse effects on the mice, as assessed by body weight (Online Supplementary Figure S4A) or analysis of $\mathrm{PB}$ cell counts (Online Supplementary Table S1). Mean trough serum concentration of CCX9588 achieved in vivo was $328 \mathrm{nM}$ (range, 76.8-886 nM; Online Supplementary Figure S4B).

In mice bearing OPM2-EV-1 or RPMI-8226-luc cells, primary tumor burden was unaffected by CCX9588 treatment (OPM2-EV-1: $P=0.91$, Figure 7A; RPMI-8226-luc: $P=0.49$, Figure $7 \mathrm{~B})$. Consistent with the effect of CCR1 KO in OPM2 cells, we observed a $66 \%$ decrease in the mean number of circulating tumor cells in the OPM2-EV-1 model $(P<0.0001$; Figure 7C); while the decrease in circulating tumor cells in the RPMI-8226-luc model did not reach statistical significance $(P=0.09$; Figure 7D). CCX9588 treatment significantly reduced dissemination to the bone, with a $22 \%$ and $70 \%$ reduction in mean tumor burden in the BM of the contralateral limb in the OPM2-EV-1 (Figure 7E) and the RPMI-8226-luc models, respectively, compared with controls $(P<0.0001$; Figure $7 \mathrm{~F})$. Furthermore, the degree of
A

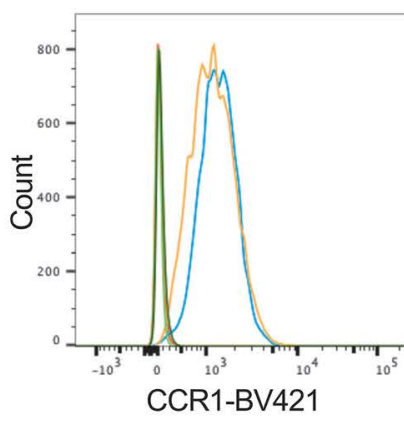

C

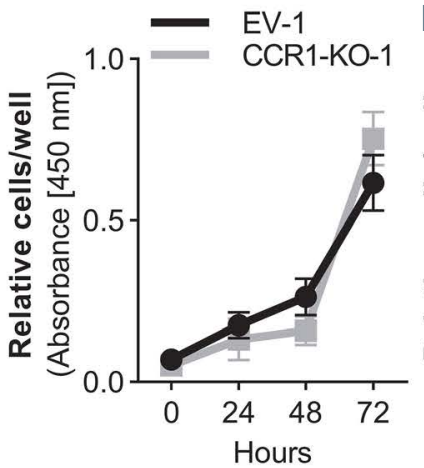

Isotype

EV-1

EV-2

CCR1-KO-1

CCR1-KO-2
B

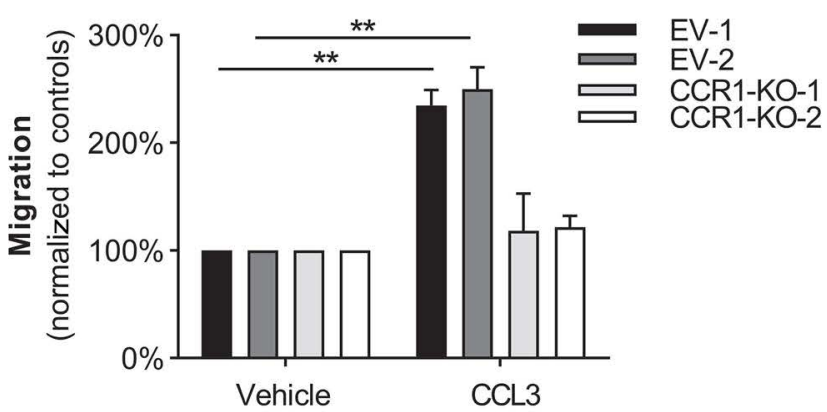

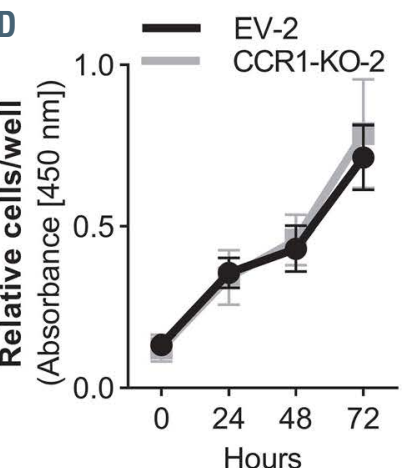

$\mathrm{E}$
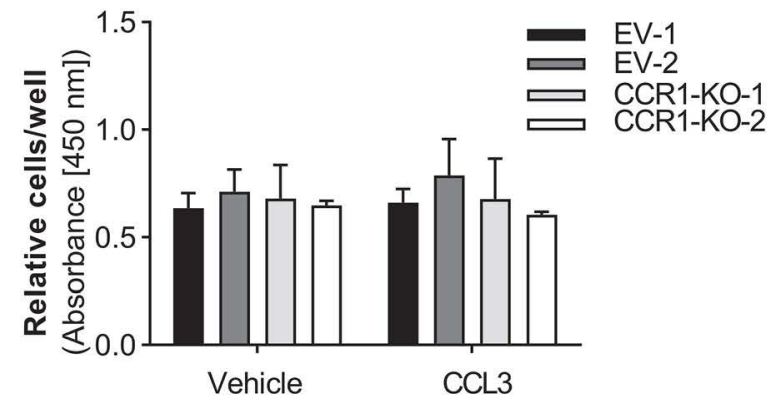

Figure 4. Knockout of CCR1 in human OPM2 multiple myeloma plasma cells decreases migration towards CCL3 and does not affect proliferation. (A) CRISPR-Cas9 mediated knockout (KO) of CCR1 was confirmed in OPM2-CCR1-KO-1 and OPM2-CCR1-KO-2 cells following staining with an anti-hCCR1 antibody or isotype control. (B) Migration of OPM2-CCR1-KO-1 and OPM2-CCR1-KO-2 cells and empty vector (EV) control cells towards $100 \mathrm{ng} / \mathrm{mL}$ rhCCL3, or media alone, was assessed after 18 hours. Migration is expressed relative to no chemoattractant controls. (C) Relative numbers of OPM2-CCR1-KO-1 or OPM2-EV-1 control cells were assessed over 72 hours. (D) Relative numbers of OPM2-CCR1-KO-2 or OPM2-EV-2 control cells were assessed over 72 hours. (E) The effect of 72 hours of treatment with 100 $\mathrm{ng} / \mathrm{mL}$ rhCCL3 on relative numbers of OPM2-CCR1-KO-1 and OPM2-CCR1-KO-2, and EV-1 and EV-2 control cells. Graphs depict mean \pm standard error of the mean of three or more independent experiments (A to E). ${ }^{*} * P<0.001$, two-way ANOVA with Sidak's multiple comparison test. 
splenomegaly in the OPM2-EV-1 model was significantly reduced compared with vehicle controls in CCX9588-treated mice $(P<0.001$; Figure $7 \mathrm{G})$. Splenomegaly was not observed in the RPMI-8226-luc model, precluding assessment of the effect of CCX9588 on splenic dissemination (Figure 7G). When treatment was delayed until 2 weeks post OPM2-EV-1 tumor cell inoculation, CCX9588-treated mice showed significantly reduced numbers of circulating tumor cells $(P<0.01$; Online Supplementary Figure S5B $)$ although delayed treatment did not significantly decrease tumor burden in the contralateral leg $(P=0.08$; Online Supplementary Figure S5C).

\section{Discussion}

MM is characterized by the presence of multiple tumors throughout the skeleton, and in some patients, soft tissues. The dissemination of MM PC is central to the progression of disease and subsequent disease relapse, highlighting the therapeutic potential of targeting key factors that regulate dissemination to delay disease progression and prevent overt relapse. While the inhibition of several factors, including selectins ${ }^{30} \mathrm{~N}$-cadherin ${ }^{31,32}$ and CXCR $4^{33}$ have been demonstrated to slow BM homing of $\mathrm{MM}$ cells in vivo, very few genes have been demonstrated to play a role in the spontaneous dissemination of MM PC from the BM. For example, overexpression of heparanase, an enzyme that cleaves heparan sulphate chains, has been reported to increase the incidence of spontaneous dissemination of
MM cells in a mouse MM xenograft model..$^{34}$ Additionally, recent data suggests that the transcription factor Twist-1 increases dissemination in an intratibial 5TGM1/KaLwRij model in vivo. ${ }^{35}$ Furthermore, as far as we are aware, no therapeutic interventions have been described that can inhibit spontaneous dissemination of MM PC in vivo. Here, our findings suggest a novel role for the chemokine receptor CCR1 in regulating the egress of MM PC from the BM to the circulation during dissemination. These findings are consistent with a role for CCR1 in metastasis in other cancer settings, with a study showing that short hairpin RNAknockdown of CCR 1 decreased migration of hepatocellular carcinoma cells in vitro and reduced the incidence of lung metastasis in vivo. ${ }^{36}$

We have previously demonstrated that hypoxia, through induction of HIF- $2 \alpha$, increases the expression of CCR1 in human MM cell lines. ${ }^{17}$ This led us to hypothesize that tumor growth in the BM exacerbates BM hypoxia, leading to increased CCR1 expression and tumor dissemination. ${ }^{17}$ Consistent with this hypothesis, our flow cytometric analysis suggests that CCR1 expression on BM PC is increased in MM patients compared with MGUS patients. In addition, our analysis suggested that elevated CCR1 expression is an independent predictor of poor overall survival in MM patients. Mechanistically, we have previously demonstrated that CCL3 treatment of human MM cell lines reduces their capacity to migrate towards exogenous CXCL12 or undergo cytoskeletal remodeling in response to CXCL12 treatment. ${ }^{17}$ Furthermore, we found that the human MM cell line U266, which does not respond to exogenous
A

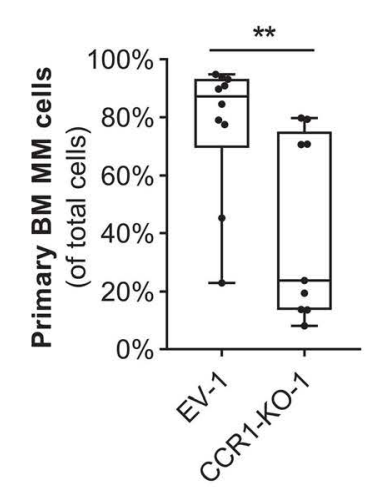

D

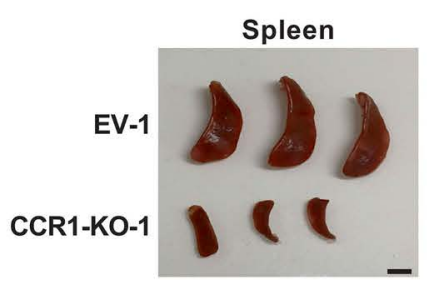

B

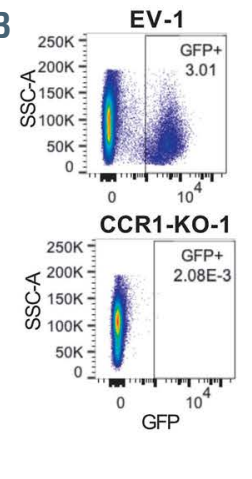

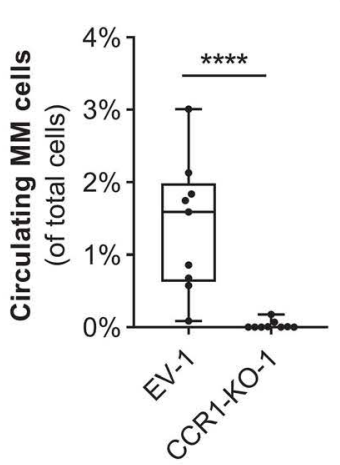

$\mathrm{E}$
C

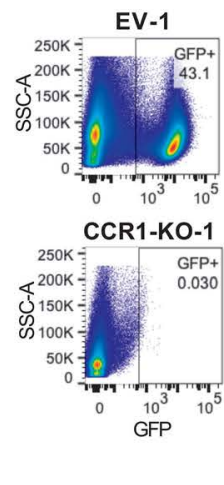

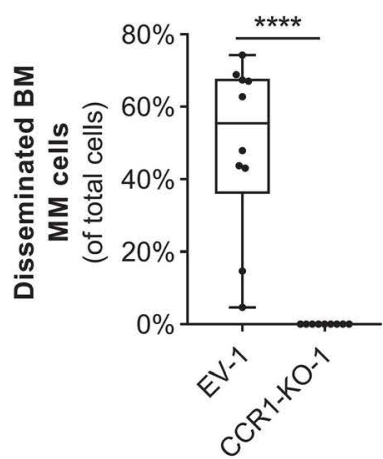

EV-1

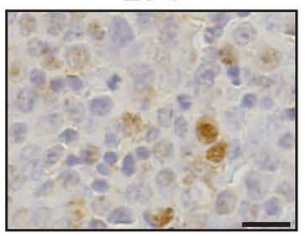

CCR1-KO-1

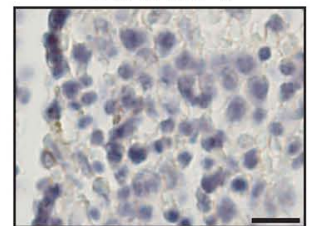

Figure 5. Dissemination of human multiple myeloma cell line OPM2 in NSG mice is abrogated by knockout of CCR1. (A) Primary tumor burden (percentage of green fluorescence protein positive [GFP+] multiple myeloma [MM] cells of total mononuclear cells) in injected tibiae after 4 weeks in NSG mice injected with OPM2-EV-1 or OPM2-KO-1 cells. (B) Number of circulating GFP+ OPM2-EV-1 or OPM2-KO-1 cells in peripheral blood of mice. (C) Tumor burden disseminated to the non-injected contralateral leg in mice injected with OPM2-EV-1 or OPM2-KO-1 cells. (D) Length of spleens collected from naïve NSG mice ( $n=7$ mice) or mice bearing OPM2-EV-1 ( $\mathrm{n}=3$ mice) or OPM2-KO-1 ( $\mathrm{n}=3$ mice) cells were measured. Image of three representative spleens from OPM2-EV-1- and OPM2-CCR1-KO-1-bearing mice. Scale bar: $10 \mathrm{~mm}$ (E) Splenic tumor cell infiltration in mice bearing OPM2-EV-1 or OPM2-CCR1-KO-1 cells was confirmed by immunohistochemistry with an anti-GFP antibody (brown). Representative flow plots of percentage of GFP+ MM cells of total mononuclear cells from one mouse per group are shown (B to C). A representative of 5 mice/group is shown; scale bar: $10 \mu \mathrm{m}(\mathrm{E})$. Box and whisker plots depict median and interquartile range, $\mathrm{n}=9-10 \mathrm{mice} / \mathrm{group}$. $* * P<0.01, * * * * P<0.0001, \mathrm{Mann}$ Whitney $\mathrm{U}$ test (A to $\mathrm{C}$ ), $* * * * P<0.0001$, one-way ANOVA with Tukey's multiple comparisons test. 
A

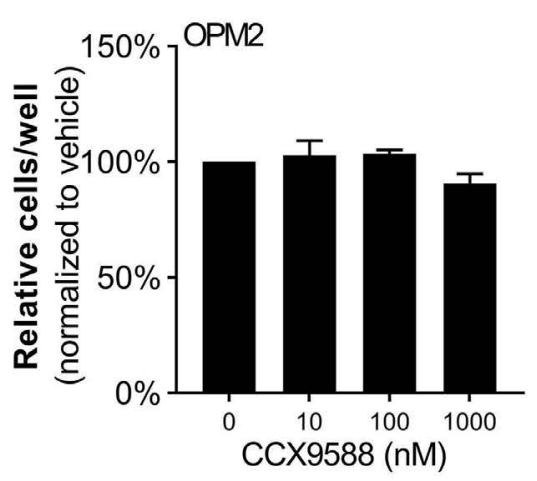

C

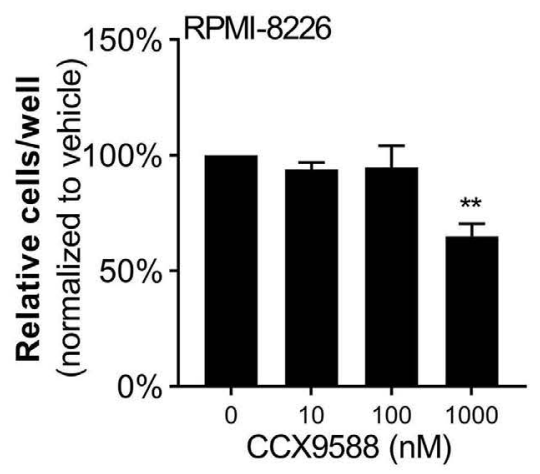

E

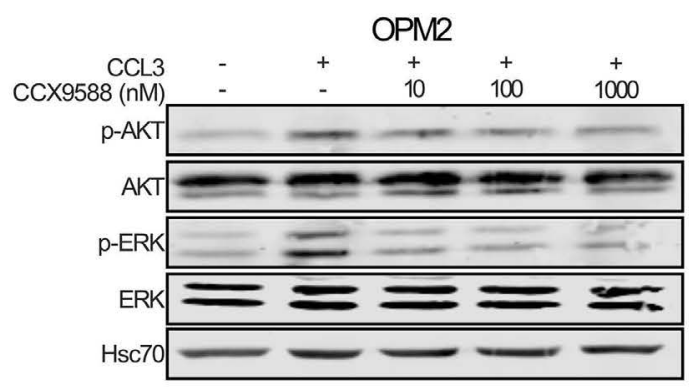

G



B

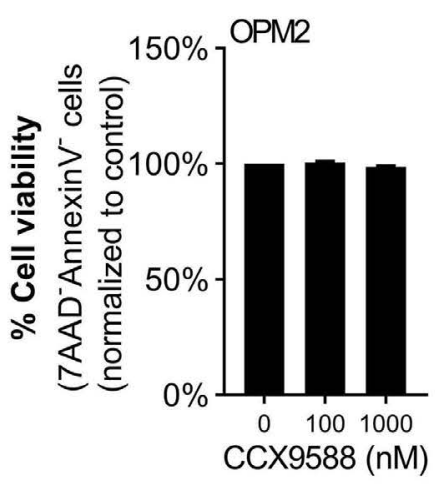

D

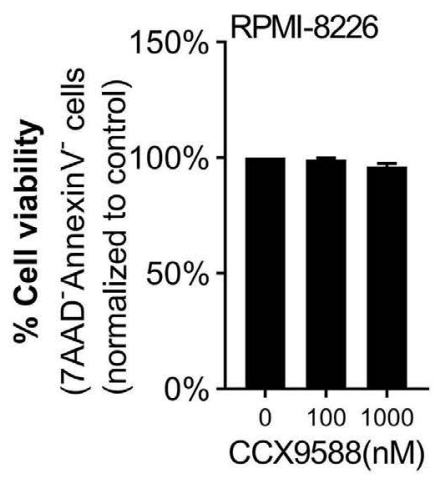

F
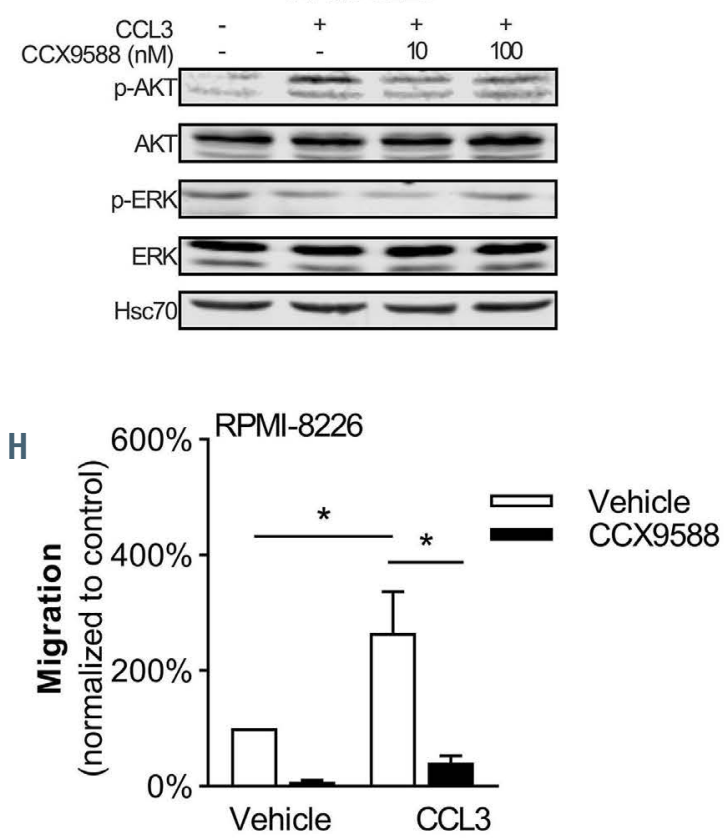

Figure 6. CCX9588 treatment prevents activation of CCR1 signaling in multiple myeloma plasma cells and their migration towards CCL3 in vitro. (A) Relative number of OPM2-EV-1 cells was assessed after 72 hours of treatment with $10 \mathrm{nM}$ to $1 \mu \mathrm{M}$ CCX9588 (all containing $0.01 \%$ dimethyl sulfoxide [DMSO)], or 0.01\% DMSO vehicle alone. (B) Viability of OPM2-EV-1 cells was assessed after 72 hours of treatment with $10 \mathrm{nM}$ to $1 \mu \mathrm{M}$ CCX9588 (all containing $0.01 \%$ DMSO), or $0.01 \%$ DMSO vehicle alone. (C) Relative number of RPMI-8226-luc cells was assessed after 72 hours of treatment with $10 \mathrm{nM}-1 \mu \mathrm{M}$ CCX9588 (all containing 0.01\% DMSO), or $0.01 \%$ DMSO vehicle alone. (D) Viability of RPMI-8226-luc cells was assessed after 72 hours of treatment with $10 \mathrm{nM}$ to 1 uM CCX9588, or $0.01 \%$ DMSO vehicle by WST-1 assay. (E) OPM2-EV-1 cells were treated with CCX9588 (10 nM to $1 \mu \mathrm{M})$ or media alone for 24 hours, and cells were stimulated with $100 \mathrm{ng} / \mathrm{mL}$ rhCCL3 for 5 minutes. Cells were lysed and western blotting was performed with antibodies against p-AKT, p-ERK1/2, total AKT and total ERK. Hsc70 was used as a loading control. A representative of three experiments is shown. (F) RPMI-8226-luc cells were treated with CCX9588 (10 nM to $1 \mu \mathrm{M})$ or media alone for 24 hours, and cells were stimulated with $100 \mathrm{ng} / \mathrm{mL}$ rhCCL3 for 5 minutes. Cells were lysed and western blotting was performed with antibodies against $\mathrm{p}$-AKT, $\mathrm{p}$-ERK1/2, total AKT and total ERK. Hsc70 was used as a loading control. A representative of two experiments is shown. (G) OPM2-EV- 1 cells were treated with $1 \mu \mathrm{M}$ CCX9588 or $0.01 \%$ DMSO vehicle control for 24 hours and migrated towards $100 \mathrm{ng} / \mathrm{mL}$ rhCCL3 or media alone. (H) RPMI-8226-luc cells were treated with $100 \mathrm{nM}$ CCX9588 or $0.01 \%$ DMSO vehicle control for 24 hours and migrated towards $100 \mathrm{ng} / \mathrm{mL}$ rhCCL3 or media alone. Graphs depict mean \pm standard error of the mean of three or more independent experiments (A to $\mathrm{D}, \mathrm{G}$ to $\mathrm{H}$ ). $* * P<0.01, * P<0.05$, one-way ANOVA with Tukey's multiple comparisons test (C) two-way ANOVA with Sidak's multiple comparisons test $(\mathrm{G}-\mathrm{H})$. 
CXCL12, produces abundant CCL3, suggesting that endogenous CCL3 expression can suppress response to CXCL12. Notably, migration of U266 cells towards CXCL12 could be restored by either CCR 1 KO or treatment with a CCR1 inhibitor. ${ }^{17}$ Here, we observed that treatment of OPM2-EV-1 control cells with CCL3 prevented migration towards CXCL12, in accordance with our previous study, ${ }^{17}$ whereas, the chemotactic response of OPM2CCR1-KO-1 cells to exogenous CXCL12 was maintained following CCL3 treatment. These data strongly suggest that CCL3/CCR1 signaling is responsible for blocking migration towards CXCL12 in these cell lines. Notably, CCL3/CCR1 signaling has been shown to drive mobilization of hematopoietic progenitors and natural killer cells from the BM in part through inactivation of CXCR4. . $^{25,26,37}$ Based on these studies, we postulated that CCL3/CCR1 signaling may inhibit the CXCL12 mediated retention of MM PC in the BM, enabling the egress of MM PC into the circulation and subsequent dissemination. Indeed, we demonstrate here that CCR1 expression increases the
A

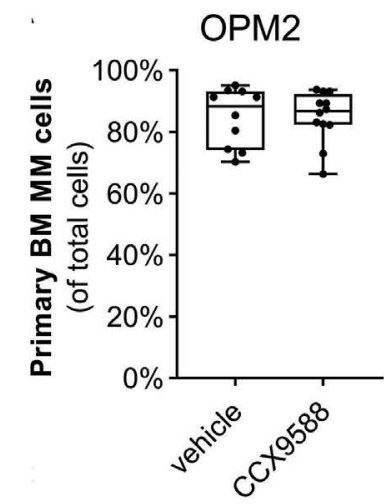

C

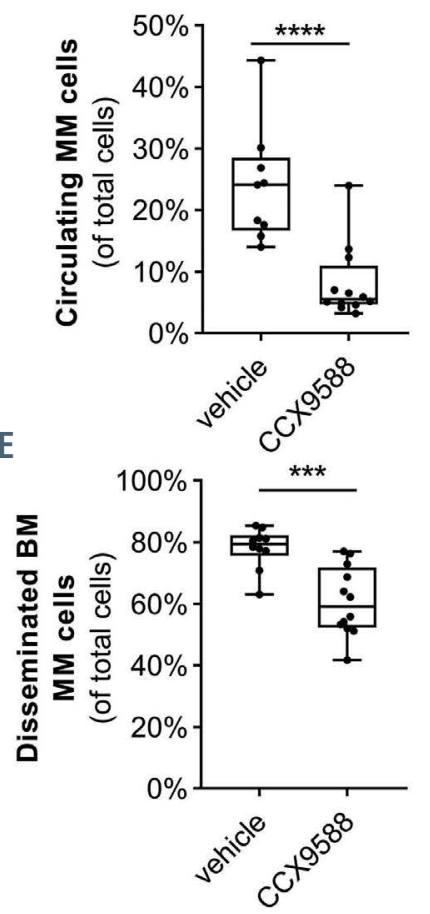

G

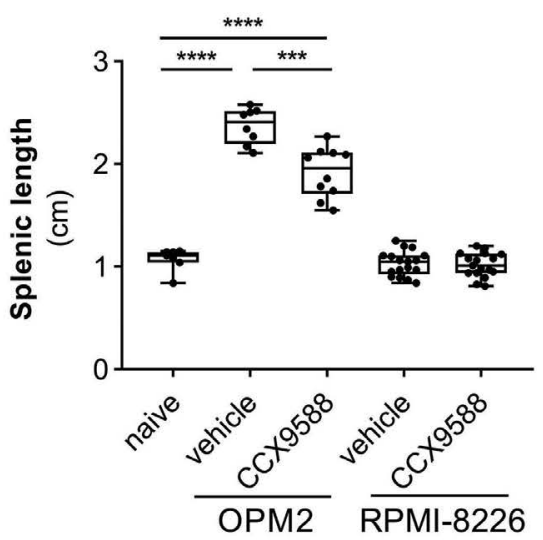

B
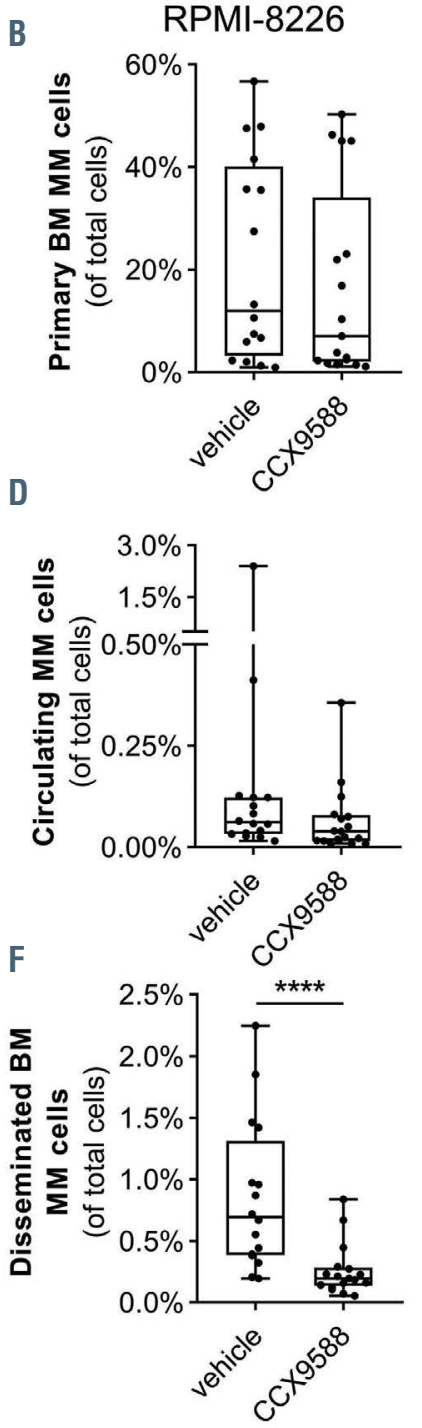

Figure 7. CCR1 inhibition reduces circulating multiple myeloma plasma cell numbers and tumor dissemination in NSG mice bearing OPM2 or RPMI-8226 cells. (A) Primary tumor burden in injected tibiae after 4 weeks in NSG mice injected with OPM2-EV-1 cells and treated days 3-28 with CCX9588 $(15 \mathrm{mg} / \mathrm{kg})$ or vehicle control at 12 hour intervals. (B) Primary tumor burden in injected tibiae after 4 weeks in NSG mice injected with RPMI-8226-Iuc cells and treated days 3-28 with CCX9588 $(15 \mathrm{mg} / \mathrm{kg})$ or vehicle control at 12-hour intervals. (C) Number of circulating OPM2-EV-1 cells in peripheral blood of mice treated days 3-28 with CCX9588 (15 mg/kg) or vehicle control at 12-hour intervals. (D) Number of circulating RPMI-8226-Iuc cells in peripheral blood of mice treated days 3-28 with CCX9588 (15 mg/kg) or vehicle control at 12-hour intervals. $(E)$. Tumor burden disseminated to the non-injected contralateral leg in mice injected with OPM2-EV-1 cells treated days 3-28 with CCX9588 (15 mg/kg) or vehicle control at 12-hour intervals. (F) Tumor burden disseminated to the non-injected contralateral leg in mice injected with RPMI-8226-luc cells treated days 3-28 with CCX9588 (15 mg/kg) or vehicle control at 12-hour intervals. (G) Spleens collected from naïve NSG mice or vehicle- or CCX9588-treated mice bearing OPM2-EV-1 or RPMI-8226luc cells were measured to assess the degree of splenomegaly. Naïve mice splenic sizes are duplicated from Figure $5 \mathrm{D}$ for comparison. Box and whisker plots depict median and interquartile range, $n=10-12$ mice/group ( $A, C$ and $E$ ), $n=17$ mice/group ( $B, D$ and $F$ ), $\mathrm{n}=7-17$ mice/group (G). $* * P<0.01, \quad * * * P<0.001$, $\star * * * P<0.0001$, Mann-Whitney test (C, E and F), ordinary one-way ANOVA with Tukey's multiple 
capacity for MM PC dissemination, while CCR1 inhibition or $\mathrm{KO}$ decreases mobilization of MM PC to the $\mathrm{PB}$, and the subsequent formation of disseminated tumors in vivo. Our findings support our hypothesis that hypoxia-mediated upregulation of CCR1 may be critical for overcoming CXCL12-mediated BM retention and enabling mobilization.

In addition to its role in counteracting CXCL12/CXCR4 signaling, CCL3 is known to act as a potent chemoattractant for murine and human MM cell lines and patientderived MM PC in vitro. ${ }^{17,20,38}$ In accordance with this, we demonstrated that CCL3 acts as a chemoattractant for OPM2 and RPMI-8226 cells, which could be blocked with CCR1 KO or inhibition. Furthermore, expression of CCR1 in 5TGM1 cells resulted in a chemotactic response to CCL3. However, while CCL3 has been shown to be produced by mesenchymal stem cell $\mathrm{s}^{20}$ and osteoclasts ${ }^{19,20}$ in the $\mathrm{BM}$, the most abundant source of CCL3 in the BM in MM patients is suggested to be the MM PC themselves..$^{18 \cdot 21,24}$ It is therefore likely that autocrine CCL3 production would interfere with the chemoattractant effect of exogenous CCL3. In further support of this, CCL3 is present at higher levels in the $\mathrm{BM}$ than in the $\mathrm{PB}$ of $\mathrm{MM}$ patients, ${ }^{21,39,40}$ suggesting that migration towards CCL3 in the $\mathrm{PB}$ would not play a significant role in the mobilization of MM PC from the BM. Instead, it is possible that autocrine CCL3 production may increase non-directional migration (chemokinesis), as has been described for chemokines CCL2 and IGF-1 in MM cell lines. ${ }^{4,42}$ In accordance with this, we found that inhibition of CCR1 in RPMI-8226-luc cells using CCX9588 resulted in a reduction in basal migration. This is consistent with a previous study, whereby the CCR1 inhibitor BX471 prevented basal migration of the human acute monocytic leukemia cell line THP-1. ${ }^{43}$ Alternatively, CCR1 has been suggested to signal without the presence of ligand and induce agonistindependent migration in some cell types. ${ }^{43}$ Decreased basal migration or chemokinesis of these cells in the presence of CCR1 inhibitor may, therefore, in part be contribute to the decrease in dissemination of RPMI-8226 and OPM2 cells observed in vivo.

We observed no effect of CCR1 expression or KO on the proliferation of $\mathrm{MM}$ cell lines in vitro in either the presence or absence of exogenous CCL3. This was despite the ability of CCL3 to induce AKT and ERK phosphorylation, which are involved in survival/proliferation pathways in $\mathrm{MM}^{24}$ This contrasts with a previous study suggesting that recombinant CCL3 increases human MM cell line proliferation in vitro. ${ }^{24}$ As such, the possibility that the relatively high serum concentration used here could be providing sufficient other growth factors to mask the effects of CCL3 cannot be excluded. Mice injected with OPM2-CCR1-KO cells had lower primary tumor burden compared with controls, suggesting that CCR1 KO may affect growth of OPM2 cells in vivo. However, we did not observe an effect of CCR1 overexpression or inhibition on primary MM tumor growth in our other in vivo models, suggesting this effect may be independent of CCR1. Additional studies are required to determine whether the retention of MM PC in the bone marrow with CCR1 KO may be causing environmental pressures, such as an increase in hypoxia, ${ }^{44}$ that is slowing their proliferation in vivo. In contrast, while CCX9588 treatment had no effect on the proliferation of OPM2 cells in vitro, we observed a decrease in the proliferation of RPMI-8226 cells with $1 \mu \mathrm{M}$ CCX9588 treatment. This contrasts with a previous study which reported that treatment with the CCR1 inhibitor CCX721 at high doses had no effect on the proliferation of RPMI-8226 cells in vitro, ${ }^{23}$ suggesting that the effects observed at high concentrations of CCX9588 here may be due to off-target effects. In support of this, CCX9588 treatment did not affect OPM2 or RPMI-8226 tumor growth in vivo. Inhibition of CCL3 or CCR1 in the murine 5T2MM and 5TGM1 models has previously been shown to decrease primary BM tumor growth, but not growth of subcutaneous tumors or cells in vitro. ${ }^{22,23}$ This suggests that CCL3/CCR1 inhibitors may affect growth factor production by cells of the BM microenvironment to indirectly affect 5TMM tumor growth. ${ }^{23}$ Similar effects were observed with osteoclast ablation using zoledronate, suggesting that these results may be secondary to decreased osteoclast activity/numbers in this model. ${ }^{23}$ The CCR1 inhibitor MLN3897 has previously been shown to decrease the pro-proliferative effects of osteoclast coculture on a CCR1-negative human MM cell line, at least in part through indirectly decreasing osteoclast IL-6 secretion, supporting the idea that effects of CCR1 inhibition on tumor growth in some in vivo models may be due to secondary effects on osteoclasts. ${ }^{19}$ However, we found no effect of CCR1 inhibition on primary tumor growth in vivo, suggesting that inhibition of microenvironmental CCR1 was not contributing to the effects observed here. Notably, we have previously demonstrated that treatment with the CXCR4 inhibitor T140 had no effect on intratibial RPMI-8226 tumor growth, despite dramatic effects on osteolysis and decreased osteoclast numbers, suggesting that inhibition of osteoclasts does not affect primary tumor growth in this model. ${ }^{45}$

Importantly, we are the first to assess the efficacy of the small molecule CCR1 inhibitor CCX9588 on dissemination in a pre-clinical model of MM. CCX9588 has been previously reported to decrease chemotaxis of $\mathrm{T}$ cells towards liver conditioned media in vitro. ${ }^{46}$ CCX9588 is an analogue of CCX354, which has previously been investigated as a therapeutic for rheumatoid arthritis in a clinical trial ${ }^{47}$ and CCX721, which has been shown to have anti-osteolytic activity in an in vivo MM model. ${ }^{23}$ While we were not able to completely prevent dissemination of MM PC using CCX9588 in OPM2 and RPMI-8226 xenograft models at this dose, these studies suggest that impeding the egress of MM PC from the BM to the PB could slow the development of disease. Further studies are required to determine whether combination therapy with other anti-myeloma agents, or more intensive treatment regimens, could achieve an enhanced effect on tumor dissemination. Notably, while both of the human MM cell lines used here do not express the alternate CCL3 receptor CCR5, CCR5 is expressed at the mRNA level in up to one third of $\mathrm{MM}$ patients (data not shown). Therefore, additional studies are warranted to determine whether CCR1 inhibition alone is sufficient to block dissemination when both CCR1 and CCR5 are expressed.

In summary, our studies have identified a novel role for the chemokine receptor CCR 1 in the context of MM pathogenesis, demonstrating that CCR1 is a key driver of MM PC egress from the $\mathrm{BM}$ to the circulation during dissemination. Furthermore, we have shown that inhibition of CCR1 via therapeutic targeting or KO can slow MM PC dissemination. Together with previous studies demonstrating that targeting of CCR1 prevents the development of severe osteolytic lesions in vivo, and our data demonstrating that CCR1 is an independent prognostic factor in MM patients, our 
study suggests that CCR1 is a potential attractive therapeutic target for MM. Future preclinical studies are warranted to investigate whether therapeutic inhibition of CCR1 has efficacy as a maintenance therapy, extending post-therapy remission and preventing overt relapse.

\section{Disclosure \\ No conflicst of interest to disclose.}

\section{Contributions}

MNZ performed experiments and wrote the manuscript; $K V$, $D R H, K M M$ and VP assisted with experiments; $K V, A C W Z$, $D R H$ and VP reviewed the manuscript; KV, ACWZ, PIC and $L B T$ designed the study; KV, ACWZ and DRH supervised the study. All authors read and approved the final manuscript.

\section{Acknowledgments}

The authors thank ChemoCentryx for generously providing the CCR1 antagonist CCX9588 for these studies. We are grateful to Vicki Wilczek and Elyse Bell for their assistance with the animal studies.

\section{Funding}

This research was supported by grant 2002138 awarded through the 2020 Priority-driven Collaborative Cancer Research Scheme (PdCCRS), co-funded by Cancer Australia and Cure Cancer, and grant 1163245 awarded through the 2018 PdCCRS, co-funded by Cancer Australia, Cure Cancer, and Leukaemia Foundation of Australia, awarded to KV. The work was partially supported by a Hans-Jürgen and Marianne Ohff Research Grant from the University of Adelaide, awarded to MZ. MZ was supported by the Florey Medical Research Foundation Doctor Chun Chung Wong and Madam So Sau Lam Memorial Postgraduate Cancer Research Top-Up Scholarship and a Short-Term Research Grant from the German Academic Exchange Service (DAAD). VP was supported by a National Health \& Medical Research Council Early Career Fellowship. KV and KMM were supported by Early Career Cancer Research Fellowships from the Cancer Council SA Beat Cancer Project on behalf of its donors and the State Government of South Australia through the Department of Health.

\section{References}

1. Rajkumar SV, Dimopoulos MA, Palumbo $\mathrm{A}$, et al. International Myeloma Working Group updated criteria for the diagnosis of multiple myeloma. Lancet Oncol. 2014;15 (12):e538-548.

2. Ghobrial IM. Myeloma as a model for the process of metastasis: implications for therapy. Blood. 2012;120(1):20-30

3. Gonsalves WI, Rajkumar SV, Gupta V, et al. Quantification of clonal circulating plasma cells in newly diagnosed multiple myeloma: implications for redefining high-risk myeloma. Leukemia. 2014;28(10):20602065.

4. Chakraborty R, Muchtar E, Kumar SK, et al. Risk stratification in myeloma by detection of circulating plasma cells prior to autologous stem cell transplantation in the novel agent era. Blood Cancer J. 2016;6(12):e512.

5. Chakraborty R, Muchtar E, Kumar SK, et al. Serial measurements of circulating plasma cells before and after induction therapy have an independent prognostic impact in patients with multiple myeloma undergoing upfront autologous transplantation. Haematologica. 2017;102(8):1439-1445.

6. Peceliunas V, Janiulioniene A, Matuzeviciene R, Zvirblis T, Griskevicius L. Circulating plasma cells predict the outcome of relapsed or refractory multiple myeloma. Leuk Lymphoma. 2012;53(4): 641-647.

7. Dingli D, Nowakowski GS, Dispenzieri A, et al. Flow cytometric detection of circulating myeloma cells before transplantation in patients with multiple myeloma: a simple risk stratification system. Blood. 2006;107(8):3384-3388.

8. Nowakowski GS, Witzig TE, Dingli D, et al. Circulating plasma cells detected by flow cytometry as a predictor of survival in 302 patients with newly diagnosed multiple myeloma. Blood. 2005;106(7):22762279.

9. Witzig TE, Gertz MA, Lust JA, Kyle RA, O'Fallon WM, Greipp PR. Peripheral blood monoclonal plasma cells as a predictor of survival in patients with multiple myeloma. Blood. 1996;88(5):1780-1787.
10. Bianchi G, Kyle RA, Larson DR, et al. High levels of peripheral blood circulating plasma cells as a specific risk factor for progression of smoldering multiple myeloma. Leukemia. 2013;27(3):680-685.

11. Kumar S, Rajkumar SV, Kyle RA, et al. Prognostic value of circulating plasma cells in monoclonal gammopathy of undetermined significance. J Clin Oncol. 2005;23(24):5668-5674.

12. Gonsalves WI, Rajkumar SV, Dispenzieri A, et al. Quantification of circulating clonal plasma cells via multiparametric flow cytometry identifies patients with smoldering multiple myeloma at high risk of progression. Leukemia. 2017;31(1):130-135.

13. Sanz-Rodríguez F, Ruiz-Velasco N, PascualSalcedo D, Teixidó J. Characterization of VLA-4-dependent myeloma cell adhesion to fibronectin and VCAM-1. Br J Haematol. 1999;107(4):825-834

14. Nie Y, Waite J, Brewer F, Sunshine MJ, Littman DR, Zou YR. The role of CXCR4 in maintaining peripheral $B$ cell compartments and humoral immunity. J Exp Med. 2004;200(9):1145-1156.

15. Azab AK, Runnels JM, Pitsillides C, et al. CXCR4 inhibitor AMD3100 disrupts the interaction of multiple myeloma cells with the bone marrow microenvironment and enhances their sensitivity to therapy. Blood. 2009;113(18):4341-4351.

16. Azab AK, Hu J, Quang P, et al. Hypoxia promotes dissemination of multiple myeloma through acquisition of epithelial to mesenchymal transition-like features. Blood. 2012;119(24):5782-5794.

17. Vandyke K, Zeissig MN, Hewett DR, et al. HIF-2a promotes dissemination of plasma cells in multiple myeloma by regulating CXCL12/CXCR4 and CCR1. Cancer Res. 2017;77(20):5452-5463.

18. Uneda S, Hata H, Matsuno $F$, et al. Macrophage inflammatory protein-1 alpha is produced by human multiple myeloma (MM) cells and its expression correlates with bone lesions in patients with $\mathrm{MM}$. $\mathrm{Br}$ J Haematol. 2003;120(1):53-55.

19. Vallet S, Raje N, Ishitsuka $K$, et al. MLN3897, a novel CCR1 inhibitor, impairs osteoclastogenesis and inhibits the interaction of multiple myeloma cells and osteo- clasts. Blood. 2007;110(10):3744-3752.

20. Moreaux J, Hose D, Kassambara A, et al. Osteoclast-gene expression profiling reveals osteoclast-derived CCR2 chemokines promoting myeloma cell migration. Blood. 2011;117(4):1280-1290.

21. Roussou M, Tasidou A, Dimopoulos MA, et al. Increased expression of macrophage inflammatory protein-1 on trephine biopsies correlates with extensive bone disease, increased angiogenesis and advanced stage in newly diagnosed patients with multiple myeloma. Leukemia. 2009;23(11):21772181.

22. Menu E, De Leenheer E, De Raeve H, et al. Role of CCR1 and CCR5 in homing and growth of multiple myeloma and in the development of osteolytic lesions: a study in the 5TMM model. Clin Exp Metastasis. 2006;23(5-6):291-300.

23. Dairaghi DJ, Oyajobi BO, Gupta A, et al. CCR1 blockade reduces tumor burden and osteolysis in vivo in a mouse model of myeloma bone disease. Blood. 2012;120(7): 1449-1457.

24. Lentzsch S, Gries M, Janz M, Bargou R, Dorken B, Mapara MY. Macrophage inflammatory protein 1-alpha (MIP-1 alpha) triggers migration and signaling cascades mediating survival and proliferation in multiple myeloma (MM) cells. Blood. 2003;101(9):3568-3573.

25. Broxmeyer HE, Hangoc G, Cooper S, Campbell T, Ito S, Mantel C. AMD3100 and CD26 modulate mobilization, engraftment, and survival of hematopoietic stem and progenitor cells mediated by the SDF1/CXCL12-CXCR4 axis. Ann N Y Acad Sci. 2007;1106:1-19.

26. Bernardini G, Sciume G, Bosisio D Morrone S, Sozzani S, Santoni A. CCL3 and CXCL12 regulate trafficking of mouse bone marrow NK cell subsets. Blood. 2008;111 (7):3626-3634.

27. Zannettino ACW, Farrugia AN, Kortesidis A, et al. Elevated serum levels of stromalderived factor-1 are associated with increased osteoclast activity and osteolytic bone disease in multiple myeloma patients. Cancer Res. 2005;65(5):1700-1709.

28. Shaughnessy JD, Jr., Qu P, Usmani S, et al. Pharmacogenomics of bortezomib test- 
CCR1 drives dissemination of multiple myeloma plasma cells

dosing identifies hyperexpression of proteasome genes, especially PSMD4, as novel high-risk feature in myeloma treated with Total Therapy 3. Blood. 2011;118(13):35123524 .

29. Hewett DR, Vandyke K, Lawrence DM, et al. DNA barcoding reveals habitual clonal dominance of myeloma plasma cells in the bone marrow microenvironment. Neoplasia. 2017;19(12):972-981.

30. Asosingh K, Günthert U, De Rave H, Van Ret I, Van Camp B, Vanderkerken K. A unique pathway in the homing of murine multiple myeloma cells: CD 44v10 medeate binding to bone marrow endothelium. Cancer Res. 2001;61(7):2862.

31. Mrozik KM, Cheong CM, Hewett D, et al. Therapeutic targeting of $\mathrm{N}$-cadherin is an effective treatment for multiple myeloma. Br J Haematol. 2015;171(3):387-399.

32. Groen RWJ, de Rooij MFM, Kocemba KA, et al. $\mathrm{N}$-cadherin-mediated interaction with multiple myeloma cells inhibits osteoblast differentiation. Haematological. 2011;96 (11): 1653 .

33. Roccaro AM, Mishima Y, Sacco A, et al. CXCR4 regulates extra-medullary myeloma through epithelial-mesenchymal-transition-like transcriptional activation. Cell Rep. 2015;12(4):622-635.

34. Yang $Y$, Macleod V, Bendre $M$, et al. Heparanase promotes the spontaneous metastasis of myeloma cells to bone. Blood. 2005;105(3):1303-1309.

35. Cheong CM, Mrozik KM, Hewitt DR, et al. Twist- 1 is unregulated by NSD2 and contributes to tumour dissemination and an epithelial-mesenchymal transition-like gene expression signature in $\mathrm{t}(4 ; 14)$-positive multiple myeloma. Cancer Lett. 2020; 475:99-108.

36. Thu Y, Gao X-M, Yang J, et al. C-C chemokine receptor type 1 mediates osteopontin-promoted metastasis in hepatocellular carcinoma. Cancer Sci. 2018;109(3): 710-723.

37. Lord BI, Woolford LB, Wood LM, et al. Mobilization of early hematopoietic progenitor cells with BB-10010: a genetically engineered variant of human macrophage inflammatory protein -1. Blood. 1995;85 (12):3412-3415.

38. Moiler C, Stromberg T, Juremalm M, Nilsson K, Nilsson G. Expression and functimon of chemokine receptors in human pultiple myeloma. Leukemia. 2003;17(1):203210.

39. Choi SJ, Cruz JC, Craig F, et al. Macrophage inflammatory protein 1-alpha is a potential osteoclast stimulatory factor in multiple myeloma. Blood. 2000;96(2):671-675.

40. Wang X-T, He Y-C, Zhou S-Y, et al. Bone marrow plasma macrophage inflammatory protein protein -1 alpha(MIP-1 alpha) and sclerostin in multiple myeloma: relationship with bone disease and clinical chiracteristics. Leuk Res. 2014;38(5):525-531.

41. Vanderkerken K, Asosingh K, Bract F, Van Ret I, Van Camp B. Insulin-like growth factor- 1 acts as a chemoattractant factor for 5T2 multiple myeloma cells. Blood. 1999;93(1):235-241.
42. Johrer K, Janke K, Krugmann J, Fiegl M, Grail R. Transendothelial migration of myeloma cells is increased by tumor necrosis factor (TNF)-alpha via TNF receptor 2 and autocrine up-regulation of MCP-1. Chin Cancer Res. 2004;10(6):1901-1910.

43. Gilliland CT, Salanga CL, Kawamura T, Trejo J, Handel TM. The chemokine receptor CCR1 is constitutively active, which leads to G protein-independent, -arrestinmediated internalization. J Biol Chem. 2013;288(45):32194-32210.

44. Mus B, de la Puente P, Arab F, Luderer M, Arab AK. Hypoxia promotes stem cell-like phenotype in multiple myeloma cells. Blood Cancer J. 2014;4(12):e262.

45. Diamond P, Labrinidis A, Martin SK, et al. Targeted disruption of the CXCL12/CXCR4 axis inhibits osteolysis in a murine model of myeloma associated bone loss. J Bone Miner Res. 2009;24(7):1150-1161.

46. Convoy MJ, Calvin KC, Kavanagh ME, et al. CCR1 antagonism attenuates T cell rafticking to omentum and liver in obesityassociated cancer. Immunol Cell Biol. 2016;94(6):531-537.

47. Tak PP, Balanescu A, Tseluyko V, et al. Chemokine receptor CCR1 antagonist CCX354-C treatment for rheumatoid arthritis: CARAT-2, a randomised, placebo controlled clinical trial. Ann Rheum Dis. 2013;72(3):337-344.

48. Chan F, Huang Y, Coll S, et al. The molecular classification of multiple myeloma. Blood. 2006;108(6):2020-2028.

haematological | 2021; 106(12)

3187 